\title{
Co-operative Spring Migration Study 1956
}

Compiled by DR. STUART HOUSTON, Yorkton

\begin{tabular}{|c|c|c|c|c|c|c|c|c|c|c|}
\hline & 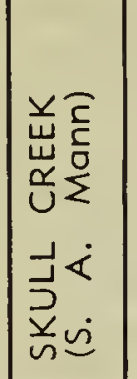 & 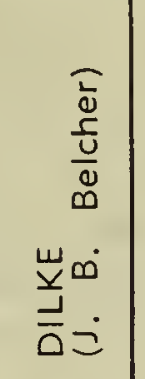 & 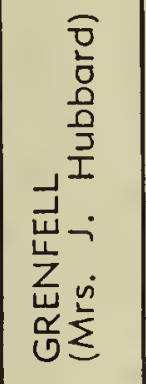 & 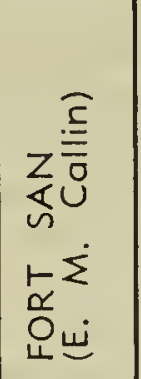 & 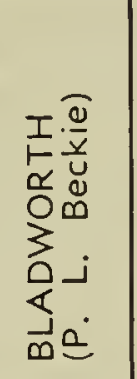 & 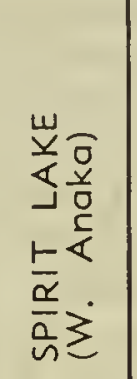 & 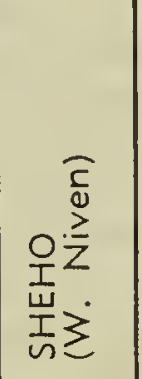 & 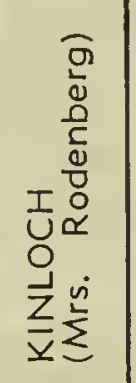 & 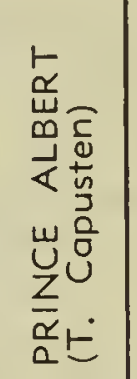 & 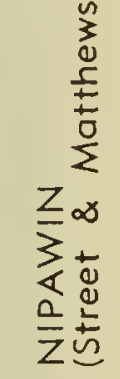 \\
\hline Whistling Swan & & Ap22 & & Apl2 & Ap 21 & My 5 & My 5 & My 8 & & \\
\hline Canada Goose & Mr21 & Ap 14 & Mr26 & Ap 8 & Apl9 & Ap 12 & Ap 4 & Apl8 & My 8 & Ap20 \\
\hline Mallard & Mr22 & Ap 1.3 & Aplo & Apl2 & Ap 9 & Apl2 & Ap 12 & Ap19 & & \\
\hline Pintail & Mr25 & -Ap 9 & Aplo & Aplo & Ap 9 & Ap 18 & $A p 12$ & Ap 19 & & \\
\hline Marsh Hawk & Mr22 & $\mathrm{Mr} 24$ & Ap 5 & Mr24 & Mr30 & Ap 18 & Ap 9 & & Ap28 & Ap 12 \\
\hline Killdeer & Mr26 & Ap 14 & Ap 17 & Apl3 & Ap 16 & Ap 18 & Ap 13 & Apl 4 & & Ap20 \\
\hline Wilson's Snipe & Ap30 & & My 1 & Ap27 & & Ap30 & & Apl 4 & & My 5 \\
\hline Mourning Dove & Ap15 & My 9 & My 12 & Ap 19 & Myl4 & My 3 & My 3 & My 2 & & My 6 \\
\hline Nighthawk & & & & My26 & My25 & Mузо & My22 & & My27 & My21 \\
\hline Rubi-throated Hummingbird & & & & My 27 & & & Je 5 & My25 & Je 4 & Je 1 \\
\hline Flicker & Ap 14 & Ap21 & Ap24 & Ap29 & Ap 19 & Ap 24 & Ap 24 & & My 6 & My 5 \\
\hline Eastern Kingbird & My21 & Myl3 & My19 & Myl9 & My20 & My20 & My21 & & & My21 \\
\hline Eastern Phoebe & & & & My 5 & & Ap20 & My17 & Ap 30 & My19 & My 9 \\
\hline Barn Swallow & My9 & My 7 & My10 & My 12 & Myl 4 & My 9 & My 9 & & & My 18 \\
\hline Purple Martin & & & My12 & & & My19 & & My 1 & & \\
\hline Crow & Mr22 & Mrit & Mr23 & Mr24 & Mr24 & Mr25 & Mr25 & Mr20 & & Ap 1 \\
\hline Catbird & My29 & & My23 & My19 & My24 & My19 & My21 & & & My17 \\
\hline Housewren & My20 & My21 & My22 & My15 & Myl6 & My21 & My21 & My24 & & Myl8 \\
\hline Brown Thrasher & Je 6 & My20 & My20 & Myl3 & My19 & My15 & My20 & & & My26 \\
\hline Red-eyed Vireo & My27 & & & My 27 & & Je 3 & My 21 & & & My28 \\
\hline Black and White Warbler & & & & My20 & & Je 3 & & My 4 & & Je 3 \\
\hline Yellow Warbler & My18 & My19 & Myl 8 & My13 & My20 & My16 & My19 & My23 & My13 & My26 \\
\hline Myrtle Warbler & My 7 & Ap 29 & & My 1 & & Ap 27 & Ap 29 & My23 & Myl3 & Ap2O \\
\hline Ovenbird & & & & & & My 18 & & & & My27 \\
\hline Redstart & & My27 & & My26 & & & & & & My28 \\
\hline Red-winged Blackbird & Ap20 & Apl 14 & Ap19 & Ap 13 & Ap 15 & Ap 19 & Ap 19 & Ap 19 & & My 4 \\
\hline Baltimore Oriole & My21 & My20 & My20 & My19 & My 20 & My 20 & My21 & My 28 & & My20 \\
\hline Rose-breasted Grosbeak & & & & My20 & & My22 & My23 & My21 & Myl9 & $\mathrm{My} 24$ \\
\hline Goldfinch & My23 & My23 & My25 & My24 & My24 & My27 & My22 & My24 & & My21 \\
\hline Slate-colored Junco & Ap 9 & Mr18 & Ap 4 & Ap 1 & Ap 3 & Ap 9 & Ap 13 & Apl 2 & My 6 & Apl 4 \\
\hline Chipping Sparrow & My 9 & My19 & & My10 & My22 & My28 & & Ap 12 & & My 12 \\
\hline White-crowned Sparrow & My 6 & My 8 & My 3 & My 6 & Ap 26 & My10 & My 18 & & & My 12 \\
\hline White-throated Sparrow & & My 8 & Myl1 & My 8 & & My 8 & My10 & My 8 & My 9 & Myl1 \\
\hline
\end{tabular}

The Co-operative Migration Study is carried out each spring all across the United States and Canada. The reports are studied in an effort to learn something of the effect of weather on migration. Last year there were reports from 20 Saskatchewan observers (Blue Jay XIII: No. 3, pp. 34-35, 1955); this year with its exceptionally late spring there were only about half that number. Perhaps this gives some measure of the effect of the weather on the bird watcher. 